\title{
PAPERS
}

\section{Physical activity and stroke in British middle aged men}

\author{
Goya Wannamethee, A G Shaper
}

\begin{abstract}
Objectives-To assess the relation between physical activity and stroke and to determine the overall benefit of physical activity for all major cardiovascular events.
\end{abstract}

Design-Prospective study of a cohort of men followed up for $\mathbf{9 . 5}$ years.

Setting-General practices in 24 towns in England, Wales, and Scotland (British regional heart study).

Subjects-7735 men aged $40-59$ at screening, selected at random from one general practice in each of 24 towns.

Main outcome measures-Fatal and non-fatal strokes and heart attacks.

Results-128 major strokes (fatal and non-fatal) occurred. Physical activity was inversely associated with risk of stroke independent of coronary risk factors, heavy drinking, and pre-existing ischaemic heart disease or stroke (relative risk 1.0 for inactivity, 0.6 moderate activity, and 0.3 vigorous activity; test for trend $p=0.008)$. The association remained after excluding men reporting regular sporting (vigorous) activity. However, vigorous physical activity was associated with a marginally significant increased risk of heart attack compared with moderate or moderately vigorous activity in men with no preexisting ischaemic heart disease or stroke (relative risk $1 \cdot 6 ; 95 \%$ confidence interval 0.96 to $2 \cdot 8$ ). In men with symptomatic ischaemic heart disease or stroke those doing moderately vigorous or vigorous activity had a risk of heart attack slightly higher than that in inactive men (relative risk $=1.6 ; 0.8$ to 3.3 ).

Conclusions-Moderate physical activity significantly reduces the risk of stroke and heart attacks in men both with and without pre-existing ischaemic heart disease. More vigorous activity did not confer any further protection. Moderate activity, such as frequent walking and recreational activity or weekly sporting activity, should be encouraged without restriction.

\section{Introduction}

Physical activity is recognised to be beneficial for ischaemic heart disease and for mortality from cardiovascular disease in general.' We recently showed that moderate and moderately vigorous physical activity, even in the absence of sporting (vigorous) activity, is sufficient to produce a beneficial effect on ischaemic heart disease in middle aged British men. ${ }^{2}$ The benefits of physical activity with respect to stroke are far less certain. The few studies that have examined this relationship specifically have mainly shown physical inactivity to be associated with an increased risk of stroke. ${ }^{3.7}$ But how much physical activity is required to obtain benefit for stroke is unclear.

This paper examines the relation between physical activity and the risk of stroke in 7735 middle aged British men drawn from general practices in 24 British towns and representative of the socioeconomic distribution of middle aged men in Great Britain. We assessed the relation in men with and without evidence of ischaemic heart disease at initial screening. In addition we examined the relation between physical activity and total cardiovascular events (stroke and heart attack) to determine the levels of physical activity associated with maximum benefit and minimum risk.

\section{Subjects and methods}

The British regional heart study is a large prospective study of cardiovascular disease comprising 7735 men aged 40-59 selected from the age-sex registers of one group general practice in each of 24 towns in England, Wales, and Scotland. The criteria for selecting the town, the general practice, and the subjects as well as the methods of data collection have been reported. ${ }^{8}$ Research nurses administered to each man a standard questionnaire that included questions on smoking habits, alcohol intake, physical activity, and medical history. Several physical measurements were made, and blood samples were taken for measuring biochemical and haematological variables. Details of smoking habits, alcohol intake, social class, blood pressure, and physical measurements have been reported. ${ }^{2}$

\section{PRE-EXISTING ISCHAEMIC HEART DISEASE OR STROKE}

The men were asked whether a doctor had ever told them that they had had angina or a myocardial infarction (heart attack, coronary thrombosis), a stroke, or several other disorders. The World Health Organisation (Rose) chest pain questionnaire for angina or possible myocardial infarction was administered to all men at the initial examination, ${ }^{9}$ and a three-orthogonal lead electrocardiogram was recorded at rest and analysed by computer in the department of medical cardiology, Glasgow Royal Infirmary.

The men were separated into three groups according to the degree of evidence of ischaemic heart disease present at screening. (1) No evidence of ischaemic heart disease on WHO (Rose) chest pain questionnaire or electrocardiogram and no recall of a doctor diagnosing ischaemic heart disease $(n=5767)$. (2) Asymptomatic ischaemic heart disease: those with electrocardiographic evidence of definite or possible myocardial ischaemia or myocardial infarction and who did not report any chest pain on WHO questionnaire or have recall of a doctor diagnosing ischaemic heart disease $(n=777)$. (3) Symptomatic ischaemic heart disease: those with angina or a possible myocardial infarction on WHO chest pain questionnaire or who remembered a doctor diagnosing angina or heart attack $(n=1165)$.

In some of the analyses those with recall of stroke $(n=40)$ have also been included in the symptomatic group (see figure). In the subsequent analyses men in 

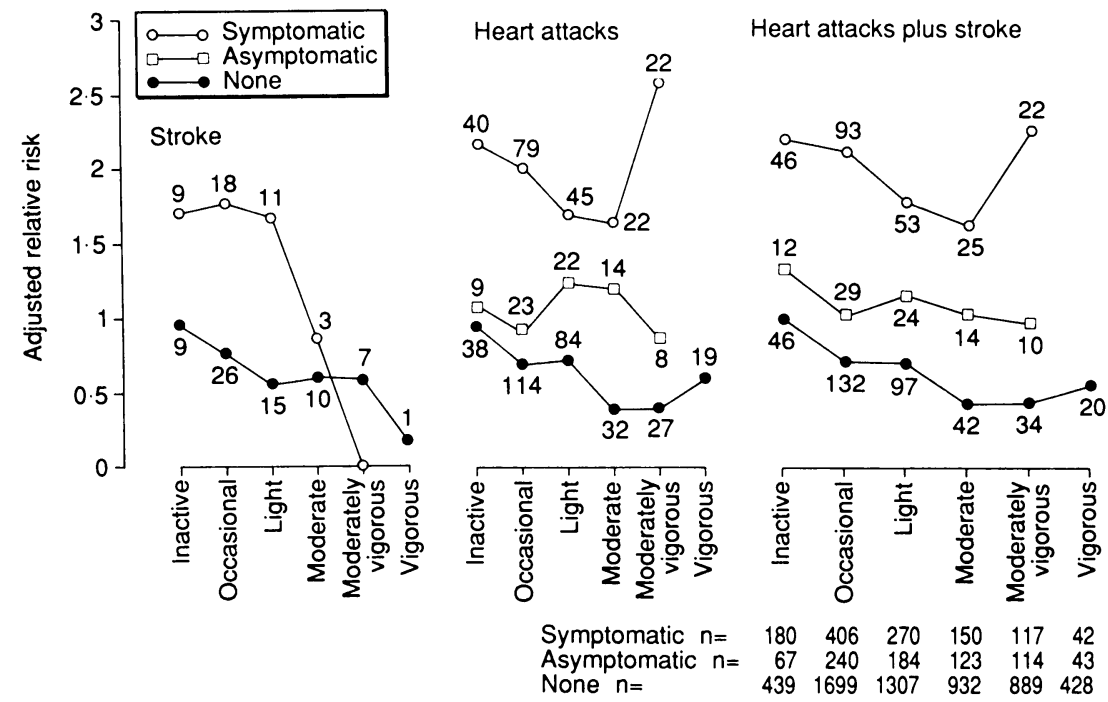

Adjusted relative risk of stroke and heart attack separately and both events combined in men with symptomatic ischaemic heart disease, asymptomatic ischaemic heart disease, and no evidence of heart disease according to physical activity. Relative risk is adjusted for age, social class, smoking, body mass index, and heavy drinking. Numbers of events are indicated on the figure

groups 2 and 3 were regarded as having pre-existing ischaemic heart disease or stroke.

\section{PHYSICAL ACTIVITY}

At screening the men were asked to indicate their usual pattern of physical activity under the headings of regular walking or cycling, recreational activity, and sporting (vigorous) activity. Regular walking and cycling related to weekday journeys, which included those to and from work. Recreational activity included gardening, walking for pleasure, and do it yourself jobs. Sporting activity included running, golf, swimming, tennis, sailing, and digging. A physical activity (exercise) score was derived for each man based on frequency and type (intensity) of the physical activity. Scores were assigned for each type of activity and duration based on the intensity and energy demands of the activities reported. This was based on the recommendations of a National Heart, Lung, and Blood Institute workshop ${ }^{10}$ and the Minnesota intensity codes. ${ }^{11}$ Full details of the derivation of the score have been described. ${ }^{2}$ The total score is a measure of how much physical activity has been carried out or energy expended.

The men were grouped into six broad categories based on their total score. The categories were inactive (score $0-2 ; \mathrm{n}=686$ ); occasional activity (score $3-5$; $\mathrm{n}=2345$ ) - regular walking or recreational activity only; light activity (score 6-8; $n=1761$ )-more frequent recreational activities or sporting exercise less than once a week; moderate activity (score $9-12 ; n=1205$ ) -cycling or very frequent recreational activities or sporting activity once a week; moderately vigorous activity (score $13-20 ; n=1120$ ) - sporting activity at least once a week or frequent cycling plus frequent recreational activities or walking or frequent sporting activity only; vigorous activity (score $\geqslant 21 ; n=513$ ) - very frequent sporting exercise or frequent sporting exercise plus other recreational activities. The physical activity score has been validated by using heart rate and lung function in men with no evidence of ischaemic heart disease. ${ }^{2}$

\section{FOLLOW UP}

All men, whether or not they had evidence of ischaemic heart disease or stroke at initial examination, were followed up for mortality from all causes and morbidity from cardiovascular causes for 9.5 years. ${ }^{12}$ Information on death was collected through the established "tagging" procedures provided by the NHS registers in Southport (England and Wales) and Edinburgh (Scotland). Non-fatal strokes were those which produced a neurological deficit that was present for more than 24 hours. Fatal episodes were those coded on the death certificate as ICD (ninth revision) 430-438. A non-fatal myocardial infarction was diagnosed according to WHO criteria. ${ }^{13}$ Fatal events were defined as deaths in which ischaemic heart disease (ICD 410-414) was the underlying cause. The ascertainment of non-fatal events (heart attack, stroke) was based on continuous reporting by general practitioners, reinforced by a regular series of reviews of all patient records at one and a half, three, five, six and a half, eight, and nine and a half years.

\section{STATISTICAL METHODS}

Multiple logistic regression was used to obtain the rates adjusted for age and the relative risks adjusted for age, smoking, body mass index, social class, heavy drinking, and systolic blood pressure. Age, body mass index, and systolic blood pressure were fitted as continuous variables, physical activity as five dummy variables, smoking as four dummy variables (never smoked, former smoker, light smoker, moderate smoker, and heavy smoker), social class as two dummy variables (manual, non-manual, and armed forces). Tests for trend were assessed by assigning quantitative values 1-6 for the six groups of physical activity and fitting physical activity as a continuous variable rather than as five dummy variables. The physical activity index was consistently used on a group basis and not as a continuous score for individuals.

\section{Results}

Within the 9.5 year follow up there were 128 major stroke events, of which 33 were fatal and 95 non-fatal, and 612 major heart attacks, of which 293 were fatal and 319 non-fatal. The physical activity score was available for 7630 men. Data on physical activity were not provided by 10 men who had a heart attack.

\section{PHYSICAL ACTIVITY AND STROKE}

Table I shows the age adjusted rates/1000 men/year for stroke in all men. Relative risks adjusted for age are also shown. There was a strong inverse association between physical activity and risk of stroke even after adjusting for age $(p<0.0001)$. In a recent report from

TABLE I-Age adjusted rates of stroke according to physical activity
Physical activity Inactive

Occasiona

Light

Moderate

Moderately vigorou

Vigorous
Men with no

ischaemic heart disease or stroke

All men

All men

Rate/1000

year

No of men No of strokes $\begin{gathered}\text { relative } \\ \text { risk) }\end{gathered}$

Rate/1000/

$439 \quad 9 \quad 2 \cdot 2$

439

$3 \cdot 1(1 \cdot 0)$

$2 \cdot 3(0 \cdot 7)$
$1 \cdot 7(0 \cdot 5)$

$1 \cdot 7(0 \cdot 5)$
$1 \cdot 4(0 \cdot 4)$

$1 \cdot 4(0.4)$
$1 \cdot 0(0.3)$

$1 \cdot 0(0 \cdot 3)$
$0 \cdot 5(0 \cdot 2)$

1699
1307
932
889

889

428

9
26
15
10
7
1

$2 \cdot 2$
1.6
1.2
1.2
0.9
0.3

$2 \cdot 2$
$1 \cdot 6$
$1 \cdot 2$
$1 \cdot 2$
$0 \cdot 9$
$0 \cdot 3$

Men with ischaemic heart disease or stroke

Rate/1000/

$\begin{array}{rrr}247 & 12 & 4 \cdot 7 \\ 646 & 26 & 4 \cdot 2 \\ 454 & 14 & 3 \cdot 3 \\ 273 & 5 & 2 \cdot 1 \\ 231 & 2 & 1 \cdot 2 \\ 85 & 1 & 1 \cdot 4\end{array}$


the British regional heart study, physical activity was shown to be strongly (inversely) associated with social class, smoking, heavy drinking, body weight, and blood pressure, ${ }^{2}$ factors known to be associated with the risk of stroke. Since blood pressure may be a mediating factor we examined the relation between physical activity and stroke adjusting first for age, social class, smoking, heavy drinking, and body mass index (table II). Even after adjustment for these factors there was a significant inverse association between physical activity and risk of stroke $(p=0 \cdot 001)$. Further adjustment for systolic blood pressure reduced the trend only slightly, and the association remained significant $(p=0 \cdot 003)$. Moderate and moderately vigorous levels of activity were associated with a $50 \%$ reduction in the risk of stroke, and the benefit from vigorous activity was even greater. Since the presence and severity of ischaemic heart disease is strongly associated with physical activity, we also adjusted for prevalence of pre-existing ischaemic heart disease or stroke. This further adjustment made little difference to the strong association seen and the trend remained significant $(\mathrm{p}=0.008)$ (table II).

\section{PHYSICAL ACTIVITY AND PRE-EXISTING ISCHAEMIC HEART DISEASE OR STROKE}

Table I shows the age adjusted rates for men with and without evidence of ischaemic heart disease or stroke. There was a significant inverse relation between physical activity and stroke in men with and without evidence of ischaemic heart disease or stroke $(p=0.01$ and $p=0.007$ respectively). Table II shows the relative risk of stroke in these two groups adjusted for age, social class, smoking, heavy drinking, and body mass index and then in addition for systolic blood pressure. After the initial adjustment the relation was similar in the two groups, although the trend was more consistent in those with ischaemic heart disease or stroke. The most vigorously active men in both groups showed more than a $60 \%$ reduction in risk of stroke. Moderate levels of physical activity were associated with a $40 \%$ reduction in those without evidence of ischaemic heart disease and a 50\% reduction in those with evidence. A test for trend gave significant results in those with ischaemic heart disease or stroke $(b=-0 \cdot 26, p=0 \cdot 02)$ and marginally significant results

TABLE II - Relative risk (95\% confidence interval) of stroke in all men and in men with and without evidence of ischaemic heart disease

\begin{tabular}{|c|c|c|c|c|c|c|c|}
\hline \multirow[b]{2}{*}{ Physical activity } & \multicolumn{3}{|c|}{$\begin{array}{c}\text { All men } \\
(\mathrm{n}=7630(128 \text { strokes }))\end{array}$} & \multicolumn{2}{|c|}{$\begin{array}{l}\text { Men with no evidence of } \\
\text { ischaemic heart disease } \\
(\mathrm{n}=5694 \text { (68 strokes)) }\end{array}$} & \multicolumn{2}{|c|}{$\begin{array}{l}\text { Men with evidence of } \\
\text { ischaemic heart disease } \\
(n=1936(60 \text { strokes }))\end{array}$} \\
\hline & A & B & $\mathrm{C}$ & A & B & A & B \\
\hline Inactive & $1 \cdot 0$ & $1 \cdot 0$ & 1.0 & $1 \cdot 0$ & $1 \cdot 0$ & $1 \cdot 0$ & $1 \cdot 0$ \\
\hline Occasion & $0.8(0.5$ to & 0.8 & 0 & $0.8(0.4$ to & 0 & $1 \cdot 0(0.4$ to & $0 \cdot 9$ \\
\hline Light & $0.6(0.3$ to 1.1$)$ & 0.6 & 0.6 & $0.6(0.2$ to 1.3$)$ & $0 \cdot 6$ & $0.7(0.3$ to 1.7$)$ & $0 \cdot 7$ \\
\hline Moderat & $0.5(0.3$ to 1.0$)$ & 0.5 & 0.6 & $0.6(0.2$ to 1.5$)$ & $0 \cdot 7$ & $0.5(0.2$ to 1.4$)$ & $0 \cdot 5$ \\
\hline Moderately vigorous & $0.4(0.2$ to 1.0$)$ & 0.5 & 0.5 & $0.6(0.2$ to 1.6$)$ & $0 \cdot 7$ & $0.3(0.1$ to 1.5$)$ & $0 \cdot 3$ \\
\hline Vigorous & $0.2(0.1$ to 0.9$)$ & $0 \cdot 2$ & $0 \cdot 3$ & $0.2(0.0$ to 1.5$)$ & $0 \cdot 2$ & $0.3(0.1$ to 3.0$)$ & $0 \cdot 4$ \\
\hline 1 est ior trena & $p=0.001$ & 0.003 & 0.008 & $p=0.06$ & $\mathrm{p}=0 \cdot 1$ & $\mathrm{p}=0.02$ & $p=0.04$ \\
\hline
\end{tabular}

$\mathrm{A}=$ Adjusted for age, social class, smoking, heavy drinking, and body mass index.

$\mathrm{B}=$ Adjusted for the factors in A plus systolic blood pressure.

$\mathrm{C}=$ Adjusted for the factors in $\mathrm{A}$ and $\mathrm{B}$ plus prevalence of ischaemic heart disease or stroke.

TABLE III - Relative risk of stroke adjusted for age, social class, smoking, body mass index, and heavy drinking and excluding men reporting sporting activity at least once a month

\begin{tabular}{|c|c|c|c|c|c|c|}
\hline \multirow[b]{2}{*}{ Physical activity } & \multicolumn{3}{|c|}{$\begin{array}{l}\text { Men with no ischaemic } \\
\text { heart disease or stroke }\end{array}$} & \multicolumn{3}{|c|}{$\begin{array}{l}\text { Men with ischaemic } \\
\text { heart disease or stroke }\end{array}$} \\
\hline & No of men & No of strokes & $\begin{array}{l}\text { Relative } \\
\text { risk }\end{array}$ & No of men & No of strokes & $\begin{array}{c}\text { Relative } \\
\text { risk }\end{array}$ \\
\hline Inactive & 439 & 9 & 1.0 & 247 & 12 & 1.0 \\
\hline Occasional & 1678 & 26 & $0 \cdot 82$ & 640 & 26 & 0.90 \\
\hline Light & 1210 & 15 & $0 \cdot 63$ & 434 & 14 & 0.75 \\
\hline Moderate & 587 & 5 & $0 \cdot 47$ & 189 & 5 & 0.67 \\
\hline Moderately vigorous & 92 & 1 & 0.53 & & & \\
\hline
\end{tabular}

in those without ischaemic heart disease or stroke $(b=-0 \cdot 17, p=0 \cdot 06)$, where $b$ is the logistic regression coefficient fitting the six physical activity index groups continuously (that is, magnitude of the trend). Further adjustment for systolic blood pressure reduced the trend slightly $(b=-0 \cdot 25$ and $b=-0 \cdot 16$, respectively).

\section{IS SPORTING ACTIVITY ESSENTIAL FOR REDUCING} STROKE?

We recently showed that sporting (vigorous) activity, although beneficial, was not essential to reduce the risk of heart attack. ${ }^{2}$ We therefore examined the relation with stroke separately in men with and without ischaemic heart disease or stroke, excluding men reporting sporting activities at least once a month $(n=2091)$. After this exclusion, the number of men with pre-existing disease in the moderately vigorous and vigorous groups was very small $(n=25)$ and these men have not been included in table III. A clear inverse trend in those with no evidence of ischaemic heart disease or stroke was evident even after adjustment for age, social class, body weight, and heavy drinking. However, this trend was not significant at the $5 \%$ level, presumably because of small numbers $(b=-0 \cdot 23$, $\mathrm{p}=0 \cdot 15$ ). In men with ischaemic heart disease or stroke the inverse trend was present but less clear $(b=-0 \cdot 16$, $\mathrm{p}=0.30$ ) than it was before exclusion of men reporting sporting activities.

\section{STROKE OR HEART ATTACKS}

Although our findings indicate a clear benefit for risk of stroke from increasing levels of physical activity in men with and without ischaemic heart disease or stroke, we recently showed that the relation between physical activity and the risk of heart attack differs in men with and without ischaemic heart disease. ${ }^{2}$ The figure shows the relation between physical activity and heart attack, stroke, and both events combined in order to assess the overall benefit of physical activity for risk of a stroke or heart attack. Data for 9.5 years' follow up in all men are presented adjusted for age, social class, smoking, body mass index, and heavy drinking. Because of the small number of men with asymptomatic and symptomatic ischaemic heart disease in the vigorous activity group $(n=43$ and $n=42$ respectively) these men, seven of whom had a heart attack, have been omitted from the analysis. As there were only 19 cases of stroke in men with asymptomatic ischaemic heart disease these data have not been presented.

Heart attack-In men without pre-existing ischaemic heart attack or stroke, the risk of heart attacks decreased significantly with increasing activity up to moderate and moderately vigorous levels $(\mathrm{p}<0.001)$ but then increased for vigorous activity. The increased risk in the vigorously active group compared with the moderate and moderately vigorous groups (relative risk $=1.6 ; 95 \%$ confidence interval 0.96 to $2 \cdot 8)$ was marginally significant $(p=0 \cdot 07)$. Compared with inactive men, men doing moderate and moderately vigorous activity had about a $60 \%$ reduction in risk $(0 \cdot 42 ; 0 \cdot 3$ to 0.7 for moderate activity and $0.44 ; 0.3$ to 0.7 for moderately vigorous activity). In men with symptomatic ischaemic heart disease or stroke, the risk decreased progressively, but not significantly, up to levels of moderate activity, but men engaged in moderately vigorous activity had an increased risk compared with moderately active men $(1 \cdot 6 ; 0.8$ to $3 \cdot 3(p=0 \cdot 1))$. No clear pattern was seen in men with asymptomatic ischaemic heart disease.

Stroke-A progressive lowering of risk was seen with increasing levels of physical activity in men with and without pre-existing ischaemic heart disease or stroke.

Heart attack or stroke-When the overall risk of 
experiencing either a heart attack or a stroke was examined (figure) in men with no evidence of ischaemic heart disease or stroke, risk decreased significantly with increasing activity up to moderate and moderately vigorous levels $(p<0.001)$ and thereafter showed a slight but non-significant increase. Men with moderate and moderately vigorous activity had over a $50 \%$ reduction in overall risk of a cardiovascular event compared with inactive men $(0.44 ; 0.3$ to 0.7 for moderate activity and $0.45 ; 0.3$ to 0.7 for moderately vigorous activity). In men with asymptomatic ischaemic heart disease physical inactivity was associated with the highest risk but thereafter there was little difference in risk between physical activity groups. In men with symptomatic ischaemic heart disease or stroke the lowest risk of an event was seen in the moderate activity group. Compared with moderately active men those engaged in moderately vigorous activity had an increased risk of an event $(1 \cdot 3$; $0 \cdot 4$ to $2 \cdot 6(\mathrm{p}=0 \cdot 15))$.

\section{Discussion}

In this study of middle aged British men physical activity showed a strong inverse association with risk of stroke independent of age, social class, smoking, heavy drinking, systolic blood pressure, and pre-existing ischaemic heart disease or stroke. Although physical activity is associated with lowering of systolic blood pressure, ${ }^{2}$ this accounted for little of the lower risk seen. The inverse relation was seen for men with and without evidence of ischaemic heart disease.

\section{PREVIOUS STUDIES}

In a study of former Harvard students (all male) participation in college sports was associated with a $60 \%$ reduction in risk of fatal stroke $10-50$ years (average 30 years) later. ${ }^{3}$ No adjustments were made for other risk factors and continued physical activity was not accounted for. In a later study of these students ${ }^{5}$ men free of diagnosed coronary heart disease at age 35-74 years reported their regular physical activity and were then followed for an average of 14 years. A strong inverse association was found between reported exercise levels and death from stroke and coronary heart disease. The exercise benefit was twice as strong for stroke as for coronary heart disease. It was independent of other risk factors, and sporting participation in student days carried little or no benefit in later years.

In the Framingham study analysis based on a 14 year follow up showed an inverse relation between physical activity and risk of stroke, but the trend was not significant after adjustment for age. ${ }^{14}$ The classification of physical activity was very broad and included leisure time and occupational activity. Annual assessment of job activity in 3886 San Francisco longshoremen for 22 years showed that those with lower energy expenditure had a $62 \%$ increase in risk of stroke compared with those with higher energy expenditure after adjusting for age, smoking, and blood pressure, but the difference was not significant. In a Dutch case-control study of survivors of stroke aged 40-74 admitted to hospital and hospital based controls, subjects were categorised according to their leisure time physical activity during the greatest portion of life as little, light, or heavy. Those engaged in heavy levels of physical activity showed a significant $60 \%$ reduction in risk of stroke compared with people engaged in little physical activity. ${ }^{6}$ Other risk factors were not adjusted for. In a random sample of men and women aged 30-59 years from two counties in eastern Finland, men and women with low physical activity at work had, respectively, a $60 \%$ and $70 \%$ increase in risk of stroke compared with those with high activity after adjusting for cardio- vascular risk factors. ${ }^{7}$ Leisure time activity was not related to risk of stroke.

The size of the reduction in risk of stroke associated with physical activity seen in these studies is similar to that in our study when all men were included.

\section{VIGOROUS SPORTING ACTIVITY}

We have previously shown that regular sporting (vigorous) activity, though beneficial in its own right, is not essential to produce a beneficial effect on heart attacks. ${ }^{2}$ This also seemed to be true for stroke, and an inverse association was still seen in men both with and without pre-existing ischaemic heart disease or stroke after excluding those reporting regular sporting activity.

\section{OVERALL EFFECT ON STROKE AND HEART ATTACKS}

We observed that compared with moderate activity vigorous physical activity in men with no evidence of ischaemic heart disease or stroke and moderately vigorous activity in those with symptomatic ischaemic heart disease or stroke were associated with an increased risk of heart attacks. We could not determine whether the reduction in the number of strokes at high levels of physical activity was a direct effect of physical activity or whether it was due to competing causes. If men who are vigorously active have higher rates of heart attack, and have attacks at a younger age than is seen for stroke, then there will be fewer susceptible men at risk of stroke. However, the consistent dose response relation for stroke in men with and without ischaemic heart disease or stroke suggests that the reduction in risk of stroke is directly associated with physical activity. In men with no evidence of ischaemic heart disease or stroke, since moderate and moderately vigorous levels of activity were associated with the lowest rates of stroke and heart attacks the lower rate of stroke seen in these men are unlikely to be due to competing causes.

Since heart attacks are over four times more common than strokes the benefits of reduction in stroke at vigorous levels of physical activity must be considered in the light of the overall risk of having either a heart attack or a stroke. In all men, irrespective of the nature of pre-existing ischaemic heart disease or stroke, physical inactivity was associated with the highest risk of having a stroke or heart attack, with risk falling with increasing activity up to moderate or moderately vigorous levels (figure). In men with no ischaemic heart disease or stroke, moderate and moderately vigorous levels of physical activity were $N$ associated with over a $50 \%$ reduction. Above this level risk increased slightly, but was still lower than that for inactivity, occasional activity, and even light activity because of the marked reduction in stroke attacks. In men with symptomatic ischaemic heart disease or stroke overall benefit was not apparent in those undertaking moderately vigorous activity despite the overwhelming reduction in stroke.

The high risk of heart attacks in the inactive men with symptomatic ischaemic heart disease or stroke may partly be attributed to the fact that these men have more severe symptoms and are therefore unable to undertake physical activity. Several prospective studies have examined the relation between physical activity and overall mortality from cardiovascular 8 causes and have found a progressive decrease in risk of death from cardiovascular disease with increasing physical activity. However, the highest category of $?$ physical activity in these studies tends to be fairly broad in definition and vigorously active subjects have not always been clearly separated. ${ }^{14} 15$ In addition, men with clinical evidence of ischaemic heart disease have been excluded - that is, the men in whom vigorous 
levels of activity seemed to have the most adverse effects. Slattery et al found that those who did intense (sporting) activity did not show any more benefit in overall mortality from cardiovascular causes than those who did frequent light or moderate activities but no intense activity.

\section{CONCLUSIONS}

Moderate levels of physical activity, such as frequent regular walking plus recreational activity or sporting activity once a week, seem to be sufficient to produce a significant beneficial effect on cardiovascular risk in men both with and without evidence of ischaemic heart disease or stroke. More vigorous activity does not seem to confer any further protection and frequent sporting (vigorous) activity may be associated with an increased risk of heart attack, especially in those with symptomatic evidence of ischaemic heart disease or stroke. Such men who are contemplating or involved in regular vigorous activity should discuss their programme with an appropriate doctor. It seems reasonable, however, to recommend widespread and unrestricted participation in moderate levels of physical activity. Moderate activity is readily attainable by large sections of the population and requires no special facilities.

This study was supported by The Chest, Heart, and Stroke Association. The British Regional Heart Study is a British Heart Foundation research group and is also supported by the Department of Health and the Institute for Alcoho Studies. We thank Dr Ivan Perry, Dr Peter Whincup, and
Mrs Mary Walker for their comments in preparing this paper.

1 Berlin JA, Colditz A. A meta-analysis of physical activity in the prevention of coronary heart disease. Am f Epidemiol 1990;132:612-27.

2 Shaper AG, Wannamethee G. Physical activity and ischaemic heart disease in middle-aged British men. Br Heart f 1991;66:384-94.

3 Paffenbarger RS, Wing AL. Characteristics in youth predisposing to fatal stroke in later years. Lancet $1967 ; 7: 753-4$.

4 Paffenbarger RS, Brand RJ, Shultz R, Jung D. Energy expenditure, cigarette smoking and blood pressure level as related to death from specific diseases. Am f Epidemiol 1978;108:12-8.

5 Paffenbarger RS, Hyde RT, Wing AL, Steinmetz CH. A natural history of the

6 Herman B, Leyten ACH, van Luijk JH, Frenken CW, Op de Coul AA, Schulte BP. An evaluation of risk factors for stroke in a Dutch community. Stroke 1982;13:334

7 Salonen JT, Puska P, Tuomilehto J. Physical activity and risk of myocardial infarction, cerebral stroke and death. Am f Epidemiol 1982;115:526-31.

8 Shaper AG, Pocock SJ, Walker M, Cohen NM, Wale CJ, Thomson AG British regional heart study: cardiovascular risk factors in middle-aged men in 24 towns. BMJ 1981;283:179-86.

9 Cook DG, Shaper AG, Macfarlane PW. Using the WHO (Rose) angina questionnaire in cardiovascular epidemiological studies. Int $\mathcal{f}$ Epidemio 1989;18:607-13.

10 Wilson PWF, Paffenbarger RS, Morris JN, Havlik RJ. Assessment method for physical activity and physical fitness in population studies: report of NHLBI workshop. Am Heart F 1986;111:1177-92.

11 Taylor HL, Jacobs DR, Schucker B, Knudsen J, Leon AS, Debecker G. A questionnaire for the assessment of leisure time physical activities. A questionnaire for the asse

. Follow-up of subjects in prospective sudies in general practice. $\mathcal{F}$ R Coll Gen Pract 1984;34:365-70

13 Shaper AG, Pocock SJ, Walker M, Phillips AN, Whitehead TP, Macfarlane PW. Risk factors for ischaemic heart disease: the prospective phase of the British regional heart study. I Epidemiol Community Health 1985;39: 197-209.

14 Kannel WB, Sorlie PD. Some health benefits of physical activity. The Framingham study. Arch Intern Med 1979;139:857-61

15 Slattery ML, Jacobs DR, Nichaman MZ. Leisure time physical activity and coronary heart disease death: the US railroad study. Circulation 1989;79. 3104-311.

(Accepted 10 December 1991)
Department of

Epidemiology and

Biostatistics, University of

Limburg, PO Box 616, 6200

MD Maastricht,

Netherlands

Bart W Koes, MA, research

fellow

Lex M Bouter, PHD, associate

professor of epidemiology

Paul G Knipschild, MD

professor of epidemiology

Department of Anatomy and Embryology,

University of Limburg

Henk van Mameren, MD,

associate professor of anatomy

Department of

Physiotherapy, University

Hospital, Maastricht,

Netherlands

Alex H M Essers, PT,

research assistant

Department of

Physiotherapy, Institute of

Higher Education,

Heerlen, Netherlands

Gard M J R Verstegen, PT, practitioner

Jo P Houben, PT, practitioner

Physiotherapy and Manual Therapy Practice,

Maastricht, Netherlands

Domien M Hofhuizen, PT,

practitioner

Correspondence to:

Mr Koes.

BMF 1992;304:601-5

\title{
Randomised clinical trial of manipulative therapy and physiotherapy for persistent back and neck complaints: results of one year follow up
}

\author{
Bart W Koes, Lex M Bouter, Henk van Mameren, Alex H M Essers, Gard M J R Verstegen, \\ Domien M Hofhuizen, Jo P Houben, Paul G Knipschild
}

\section{Abstract}

Objective-To compare the effectiveness of manipulative therapy, physiotherapy, treatment by the general practitioner, and placebo therapy in patients with persistent non-specific back and neck complaints.

Design-Randomised clinical trial.

Setting-Primary health care in the Netherlands.

Patients - 256 patients with non-specific back and neck complaints of at least six weeks' duration who had not received physiotherapy or manipulative therapy in the past two years.

Interventions - At the discretion of the manipulative therapists, physiotherapists, and general practitioners. Physiotherapy consisted of exercises, massage, and physical therapy (heat, electrotherapy, ultrasound, shortwave diathermy). Manipulative therapy consisted of manipulation and mobilisation of the spine. Treatment by general practitioners consisted of drugs (for example, analgesics), advice about posture, home exercises, and (bed)rest. Placebo treatment consisted of detuned shortwave diathermy (10 minutes) and detuned ultrasound (10 minutes).

Main outcome measures-Changes in severity of the main complaint and limitation of physical functioning measured on 10 point scales by a blinded research assistant and global perceived effect measured on a 6 point scale by the patients.

Results - Many patients in the general practitioner and placebo groups received other treatment during follow up. Improvement in the main complaint was larger with manipulative therapy (4.5) than with physiotherapy (3.8) after 12 months' follow up (difference $0.9 ; 95 \%$ confidence interval 0.1 to 1.7 ). Manipulative therapy also gave larger improvements in physical functioning (difference $0.6 ;-0.1$ to 1.3 ). The global perceived effect after six and 12 months' follow up was similar for both treatments.

Conclusions-Manipulative therapy and physiotherapy are better than general practitioner and placebo treatment. Furthermore, manipulative therapy is slightly better than physiotherapy after 12 months.

\section{Introduction}

In most cases of back pain and neck pain no underlying disease can be established and the causes of the complaints remain unknown. ${ }^{12}$ Fortunately, most patients with acute complaints recover within a few weeks, often with the help of (bed)rest, analgesics, and advice about posture and exercises. ${ }^{3}$ The complaints disappear within a few months in about $90 \%$ of the cases, ${ }^{245}$ although the recurrence rate is high. ${ }^{1}$ When the complaints persist there are several options for treatment. General practitioners in the Netherlands often refer patients with persisting complaints for physiotherapy or, less commonly, for manipulative therapy.

Physiotherapists usually give exercise therapy, alone or in combination with other treatments - for example, 